\title{
Psychological outcomes and surgical decisions after genetic testing in women newly diagnosed with breast cancer with and without a family history
}

\author{
Bettina Meiser ${ }^{1}$. Veronica F. Quinn ${ }^{1} \cdot$ Gillian Mitchell $^{2,3} \cdot$ Kathy Tucker $^{4} \cdot$ Kaaren J. Watts $^{1} \cdot$ Belinda Rahman $^{1}$. \\ Michelle Peate $^{1,5} \cdot$ Christobel Saunders $^{6} \cdot$ Elizabeth Geelhoed $^{7} \cdot$ Margaret Gleeson $^{8} \cdot$ Kristine Barlow-Stewart $^{9}$. \\ Michael Field ${ }^{10} \cdot$ Marion Harris $^{11} \cdot$ Yoland C. Antill ${ }^{12} \cdot$ Rachel Susman $^{13} \cdot$ Michael T. Bowen $^{14,15} \cdot$ Llew Mills $^{1} \cdot$ \\ Judy Kirk ${ }^{16,17}$ for the TFGT Collaborative Group
}

Received: 11 July 2017 / Revised: 9 November 2017 / Accepted: 18 November 2017 / Published online: 30 March 2018

(c) European Society of Human Genetics 2018

\begin{abstract}
In patients with early breast cancer, personal and tumour characteristics other than family history are increasingly used to prompt genetic testing to guide women's cancer management (treatment-focused genetic testing, 'TFGT'). Women without a known strong family history of breast and/or ovarian may be more vulnerable to psychological sequelae arising from TFGT. We compared the impact of TFGT in women with $(\mathrm{FH}+)$ and without $(\mathrm{FH}-)$ a strong family history on psychological adjustment and surgical decisions. Women aged $<50$ years with high-risk features were offered TFGT before definitive breast cancer surgery and completed self-report questionnaires at four time points over 12 months. All 128 women opted for TFGT. TFGT identified 18 carriers of a disease-causing variant $(50.0 \% \mathrm{FH}+)$ and 110 non-carriers $(59.1 \% \mathrm{FH}+)$. There were no differences based on family history in bilateral mastectomy (BM) uptake, $p=.190$, or uptake of risk-reducing bilateral salpingo-oophorectomy (RRBSO), $p=.093$. FH - women had lower decreases in anxiety a year after diagnosis, $p=.011$, and regret regarding their decision whether to undergo $\mathrm{BM}, p=.022$, or RRBSO, $p=.016$ than $\mathrm{FH}+$ women. FH - carriers reported significantly higher regret regarding their TFGT choice $(p=.024)$ and test-related distress $(p=.012)$ than $\mathrm{FH}+$ carriers, but this regret/distress could not be attributed to a concern regarding a possible worse prognosis. These findings indicate that $\mathrm{FH}$ - women may require additional counselling to facilitate informed decisions. Carriers without a family history may require additional follow-up counselling to facilitate psychological adjustment to their positive variant results, extra support in making surgical decisions, and counselling about how best to communicate results to family members.
\end{abstract}

\section{Introduction}

Traditionally, women with breast cancer with a strong family history of breast and/or ovarian cancer are referred to a familial cancer clinic, or similar, for consideration of genetic testing following completion of surgery and adjuvant chemo-

The additional members of the Treatment Focused Genetic Testing Collaborative Group are listed at the end of this paper.

Bettina Meiser and Veronica F. Quinn contributed equally to this work.

Bettina Meiser

b.meiser@unsw.edu.au

Extended author information available on the last page of the article and/or radiotherapy. Increasing evidence suggests that knowledge of whether a disease-causing variant is present may influence decisions about immediate breast cancer management [1], and thus treatment-focused genetic testing (TFGT) following a new diagnosis of breast cancer is becoming increasingly widespread. In women with a BRCAI or $B R C A 2$ disease-causing variant, the 10 -year risk of developing a new primary cancer in the ipsilateral breast is estimated at $12.9 \%$ (95\% CI, 8.7-17.1) [2]. In women diagnosed with invasive breast cancer under 50 years of age, the 10-year risks for a contralateral second primary cancer are $21.1 \%$ (95\% CI, 15.4-27.4) for BRCAl, and 10.8\% (95\% CI, 4.7-19.6) for $B R C A 2$, variant carriers compared with $5.1 \%$ (95\% CI, 4.5-5.7) for non-carriers, with a younger age at diagnosis of the first breast cancer a significant predictor of higher risk [3]. A confirmed BRCA1 or BRCA2 pathogenic variant may facilitate a decision between breast conservation 
followed by radiation or more radical surgery with both a therapeutic and risk-reducing intent. For carriers of a BRCAl/ 2 pathogenic variant, contralateral mastectomy has been shown to lead to a significant reduction in breast cancer related mortality $[4,5]$. Carriers may also consider riskreducing bilateral salpingo-oophorectomy (RRBSO) to reduce their risk of ovarian and tubal cancers [6] and (for BRCA2) breast cancer [7]. Knowledge of whether a pathogenic variant is present may also influence choice of adjuvant chemotherapy and other targeted agents [8].

Family history has traditionally been the major selection criteria for genetic testing, but this has significant limitations as $30-50 \%$ of carriers of a BRCAl/2 pathogenic mutation do not report a significant family history [1], due to small family size, few female relatives, patrilineal inheritance, lower than predicted cancer penetrance, nondisclosure of relevant family cancer diagnoses or isolation from family members $[9,10]$. Consequently, characteristics other than a strong family history are increasingly being used to select women for genetic testing, including triplenegative receptor status of the breast tumour, presence of bilateral breast cancer, and ethnic groups with prevalent founder variants. These expanded selection criteria, together with the introduction of multi-gene panel testing [11], mean that an increasing number of women with no known or minimal family history will be offered genetic testing.

The impact of genetic testing in women with cancer and a strong family history is well documented [12, 13]. Studies have found that distress among carriers increases shortly after receiving results and returns to pretesting levels over time [12,13], and testing shortly after a cancer diagnosis does not increase distress [14, 15]. Very few studies are currently available on the psychological impact of TFGT or traditional genetic testing for BRCAl/2 variants in the subgroup of women with no knowledge of or minimal family history, and the available studies are limited to Ashkenazi Jewish people undergoing population-based screening [16, 17]. It has been argued that people participating in population-based genetic testing expect negative results (i.e., no variant to be found), and those known to be at risk based on family history expect positive results [18]. Accordingly, women with a strong family history who undergo TFGT are more likely to be aware that they are at risk of carrying a pathogenic variant and may experience lower levels of distress if identified as carriers, compared to those with no/ minimal known family history. Women with no/minimal family history may therefore be particularly vulnerable to adverse psychological sequelae arising from TFGT. Hence, there is an imperative to understand the psychological outcomes of TFGT in this group of women. This article describes the impact of TFGT on psychological adjustment and surgical decisions in women with no/minimal family history compared to women with a strong family history.

\section{Materials and methods}

\section{Participants}

The data were collected as part of a non-inferiority trial, which examined the impact of replacing one-on-one pre-test genetic counselling with brief educational materials [19] and found no difference between women based on pre-test counselling method [20]. Women were recruited through the breast clinics associated with eight participating familial cancer clinics located in three Australian states. All women aged between 18 and 49 years diagnosed with early invasive breast cancer or ductal carcinoma in-situ were eligible if they were proficient in English and had either: (i) a strong family history of breast and/or ovarian cancer (denoted $\mathrm{FH}+$ ), or (ii) no known or a minimal family history of breast and/or ovarian cancer (denoted $\mathrm{FH}-$ ) but a personal diagnosis of bilateral breast cancer, Ashkenazi Jewish ancestry and/or triple-negative breast cancer. The categorisation of women as having either a strong family history or a minimal family history was done according to national guidelines [21]. In particular, women were categorised as having a strong family history if they had a) 3 or more affected relatives on one side of the family, including the patient OR if they had b) 2 or more affected relatives including the patient on one side of the family plus a high-risk feature (diagnosis $<40$ years, bilateral breast cancer, breast and ovarian cancer in the same woman, Ashkenazi Jewish ancestry, or male breast cancer). Women were excluded if they had already had a therapeutic mastectomy or breast radiotherapy for their current cancer or genetic counselling and/or testing, diagnosis of distant metastases or lobular carcinoma in-situ only.

Eligible women were invited to participate by their treating breast surgeon and were informed they would be able to choose whether or not to have TFGT. TFGT involved germline BRCA1 and BRCA2 testing by Sanger sequencing and Multiplex Ligation-dependent Probe Amplification, with a turnaround time of eight working days from blood draw to result disclosure, at no cost to the patient. All participants received their test results in a faceto-face appointment at the familial cancer clinic attached to their treating hospital.

\section{Measures}

Women completed four questionnaires over a period of 12 months at the following times: (1) at baseline following recruitment by their surgeon, (2) 1 week after receiving brief educational materials or face-to-face genetic counselling, (3) 2 weeks following disclosure of the TFGT result, and (4) 12 months after enrolment. The following measures were included in patient questionnaires:

Sociodemographic data: were collected at baseline. 


\section{Impact of event scale (IES)}

Fifteen items measured the frequency and severity of breastcancer-specific worry (range 0 to 75 , with higher scores indicating more worry) [22]. The IES was administered at all time points.

\section{Hospital anxiety and depression scale (HADS)}

The 14-item HADS has two subscales measuring anxiety and depression [23]. Scores range from 0 to 21 for each subscale, with higher scores indicating more anxiety or depression. The HADS was administered at all time points.

\section{Test-related distress and positive experiences}

Six items assessed distress (range 0-30) and four items positive experiences (range 0-30) about genetic testing [24], with higher scores indicating more distress and positive experiences. This measure was administered 2-weeks post-testing and 12-months post-enrolment.

The following measures were administered 12-months post-enrolment:

\section{Uptake of BM or RRBSO}

One item asked women whether they had a BM or RRBSO since their diagnosis of breast cancer. Response options included: "Yes, I had a BM/RBBSO", "No, I did not have a $\mathrm{BM} / \mathrm{RBBSO}$ and I do not intend to have one" and "No, I did not have a BM/RBBSO but I do intend to have one". For women with missing self-report data on BM/RRSBO, surgery decisions were ascertained through medical files.

\section{Decision regret scale (DRS) - Genetic testing choice}

The five-item DRS was adapted to measure regret in relation to the decision to undergo TFGT [25]. Higher scores indicate more regret (range 0-100).

\section{DRS - surgery choice}

Women completed two different versions of the DRS to measure decision regret relating to $\mathrm{BM}$ and RRBSO.

\section{Clinical information}

was collected directly from the surgical teams and/or pathology reports. We combined the data concerning tumour size and grade, nodal involvement, oestrogen and progesterone receptor status, and ductal carcinoma in-situ vs. invasive cancer into one prognostic variable (favourable, intermediate, unfavourable) guided by published criteria
[26] to reduce multicollinearity between clinical variables, while still capitalising on the unique variance contributed by each. Hereafter, this variable will be referred to as 'prognostic status', and it was entered into all equations to control for potential differences in prognostic characteristics between those with and without a family history.

\section{Data analysis}

Simple linear regression was used to assess outcome variables with one time point. For outcome variables with more than one time point, linear mixed effects models were used. In all models family history, presence of a pathogenic variant and time point (if relevant to the variable) were included, as was the participant's randomisation status from the original study and their prognostic status. Two-way interactions between family history, presence of a pathogenic variant and time point were also examined but were only included in the final model if they were statistically significant. Linear mixed effects models used first-order autoregressive covariance structure, as it has been shown to be more suitable for modelling growth processes and showed superior goodness of fit (Akaike and Bayesian Information Criteria) to both unstructured and standard parabolic error covariance structures [27]. Simple coding was used on family history, presence of a pathogenic variant, and time point variables to generate interpretable estimates of main effects within the framework of hierarchical linear and standard linear regression. Two categorical outcomes, decision to undergo bilateral mastectomy (BM) and RRBSO, were analysed using binary logistic regression, where age was added due to its relevance to surgical decision-making. On the basis of clinical considerations, it was decided that for the BM analysis, age was entered as a continuous variable, and for RRBSO we instead used a dichotomised version with women classified as aged $\leq 40$ or $>40$ years due to the importance of age 40 as a threshold for recommendation of RRBSO in Australia [28]. All analyses were conducted in R v3.3.0 and results were considered statistically significant when $p<.05$.

\section{Results}

Patient recruitment took place from July 2010 to October 2012. Sociodemographic, medical and family history characteristics of the sample are detailed in Table 1. Figure 1 shows the flow of participants through the study. Of the 128 participants included in the analyses, 107 (83.5\%) also returned the 12-months questionnaire. No significant differences were found between participants who completed all questionnaires and those who did not in terms of baseline 
psychological characteristics as well as age and educational level. Seventy-four (57.8\%) women were eligible on the basis of strong family history, while the remaining had no known or only minimal family history and were recruited based on the other risk factors listed above. Apart from $\mathrm{FH}-$ women being more likely to have Grade 3 and oestrogen receptor positive tumours and a better prognostic status than $\mathrm{FH}+$ women, there were no significant differences for the collected variables

Table 1 Sociodemographic, medical and family history characteristics of usual care and intervention group $(N=128)$

\begin{tabular}{|c|c|c|c|c|c|}
\hline Variables & & $\begin{array}{l}\text { Strong family history of } \\
\text { breast cancer }(N=74) \\
\text { Mean }(\mathrm{SD})\end{array}$ & $\begin{array}{l}\text { No/minimal family history } \\
\text { of breast cancer }(N=54) \\
\text { Mean (SD) }\end{array}$ & $\begin{array}{l}\text { Total sample } \\
(N=128) \\
\text { Mean }(\mathrm{SD})\end{array}$ & $p$ \\
\hline Age (diagnosis) & Range: 27,49 & $40.1(5.6)$ & $40.5(5.9)$ & $40.3(5.7)$ & 0.69 \\
\hline \multirow[t]{2}{*}{ Boadicea score ${ }^{a}$} & $B R C A 1 / 2$ (range: $0.5,80.5$ ) & $14.5(20.1)$ & $11.1(14.3)$ & $13.1(17.9)$ & 0.29 \\
\hline & Level & $N(\%)$ & $N(\%)$ & $N(\%)$ & \\
\hline \multirow[t]{2}{*}{ Children } & Yes & $58(78.4)$ & $40(74.1)$ & $98(76.6)$ & 0.57 \\
\hline & No & $16(21.6)$ & $14(25.9)$ & $30(23.4)$ & \\
\hline \multirow{4}{*}{$\begin{array}{l}\text { Highest education level } \\
\text { achieved }\end{array}$} & No high school certificate & $7(9.5)$ & $5(9.3)$ & $12(9.4)$ & 0.88 \\
\hline & High school certificate & $10(13.5)$ & $10(18.5)$ & $20(15.6)$ & \\
\hline & Vocational college/college & $22(29.7)$ & $14(25.9)$ & $36(28.1)$ & \\
\hline & Degree/postgraduate degree & $35(47.3)$ & $25(46.3)$ & $60(46.9)$ & \\
\hline \multirow[t]{2}{*}{ Marital status } & Married/living as married & $49(66.2)$ & $38(70.4)$ & $87(68.0)$ & 0.62 \\
\hline & Not married & $25(33.8)$ & $16(29.6)$ & $41(32.0)$ & \\
\hline \multirow{2}{*}{$\begin{array}{l}\text { Language spoken at } \\
\text { home }\end{array}$} & English & $71(95.9)$ & $50(92.6)$ & $121(94.5)$ & 0.41 \\
\hline & $\begin{array}{l}\text { Language other than } \\
\text { English }\end{array}$ & $3(4.1)$ & $4(7.4)$ & $7(5.5)$ & \\
\hline \multirow[t]{2}{*}{$\begin{array}{l}\text { Uni- vs bilateral breast } \\
\text { cancer, }, \text {, c }\end{array}$} & $\begin{array}{l}\text { Unilateral breast cancer } \\
\text { Bilateral breast cancer }\end{array}$ & $71(97.3)$ & $46(85.2)$ & $117(92.1)$ & 0.01 \\
\hline & & $2(2.7)$ & $8(14.8)$ & $10(7.9)$ & \\
\hline \multirow[t]{2}{*}{ Carrier status } & $\begin{array}{l}\text { Carrier of pathogenic } \\
\text { variant }\end{array}$ & $9(12.2)$ & $9(16.7)$ & $18(14.1)$ & 0.47 \\
\hline & $\begin{array}{l}\text { No pathogenic variant } \\
\text { found }\end{array}$ & $65(87.8)$ & $45(83.3)$ & $110(85.9)$ & \\
\hline \multirow{2}{*}{$\begin{array}{l}\text { Invasive vs ductal } \\
\text { carcinoma in situ }\end{array}$} & Invasive carcinoma & $67(90.5)$ & $54(100.0)$ & $121(94.5)$ & 0.020 \\
\hline & $\begin{array}{l}\text { Ductal carcinoma in situ } \\
\text { only }\end{array}$ & $7(9.5)$ & $0(0.0)$ & $7(5.5)$ & \\
\hline \multirow[t]{2}{*}{ Tumour size, ${ }^{\mathrm{c}, \mathrm{d}}$} & $\leq 2 \mathrm{~cm}$ & $35(53.0)$ & $30(55.6)$ & $65(54.2)$ & 0.78 \\
\hline & $>2 \mathrm{~cm}$ & $31(47.0)$ & $24(44.4)$ & $55(45.8)$ & \\
\hline \multirow[t]{3}{*}{ Tumour grade ${ }^{c, d}$} & 1 & $5(7.7)$ & $4(7.4)$ & $9(7.6)$ & 0.001 \\
\hline & 2 & $28(43.1)$ & $7(13.0)$ & $35(29.4)$ & \\
\hline & 3 & $32(49.2)$ & $43(79.6)$ & $75(63.0)$ & \\
\hline \multirow[t]{2}{*}{ Nodal Involvement ${ }^{\mathrm{c}, \mathrm{d}}$} & Yes & $27(43.5)$ & $17(32.1)$ & $44(38.3)$ & 0.21 \\
\hline & No & $35(56.5)$ & $36(67.9)$ & $71(61.7)$ & \\
\hline \multirow{2}{*}{$\begin{array}{l}\text { Oestrogen receptor } \\
\text { status }\end{array}$} & Positive & $54(80.6)$ & $14(25.9)$ & $68(56.2)$ & $<0.001$ \\
\hline & Negative & $13(19.4)$ & $40(74.1)$ & $53(43.8)$ & \\
\hline \multirow[t]{3}{*}{ Prognostic status } & Favourable & $16(21.6)$ & $4(7.4)$ & $20(15.6)$ & $<0.001$ \\
\hline & Intermediate & $25(33.8)$ & $7(13.0)$ & $32(25.0)$ & \\
\hline & Unfavourable & $33(44.6)$ & $43(80.0)$ & $76(59.3)$ & \\
\hline
\end{tabular}

${ }^{a}$ Breast and Ovarian Analysis of Disease Incidence and Carrier Estimation Algorithm (BOADICEA) [25]

${ }^{\mathrm{b}}$ On the basis of pathology records, rather than surgeon's report

${ }^{\mathrm{c}}$ Varying cell sizes due to the missing data

${ }^{\mathrm{d}}$ Tumour size and grade and nodal involvement only relevant to invasive carcinoma 
Fig. 1 Flow of participants through the study $\mathrm{RC}$ research co-ordinator; $\mathrm{T} 1=$ baseline questionnaire; $\mathrm{T} 2=$ within 1 week of receiving education; $\mathrm{T} 3=2$ weeks post-disclosure of testing result; $\mathrm{T} 4=12$ months after study enrolment

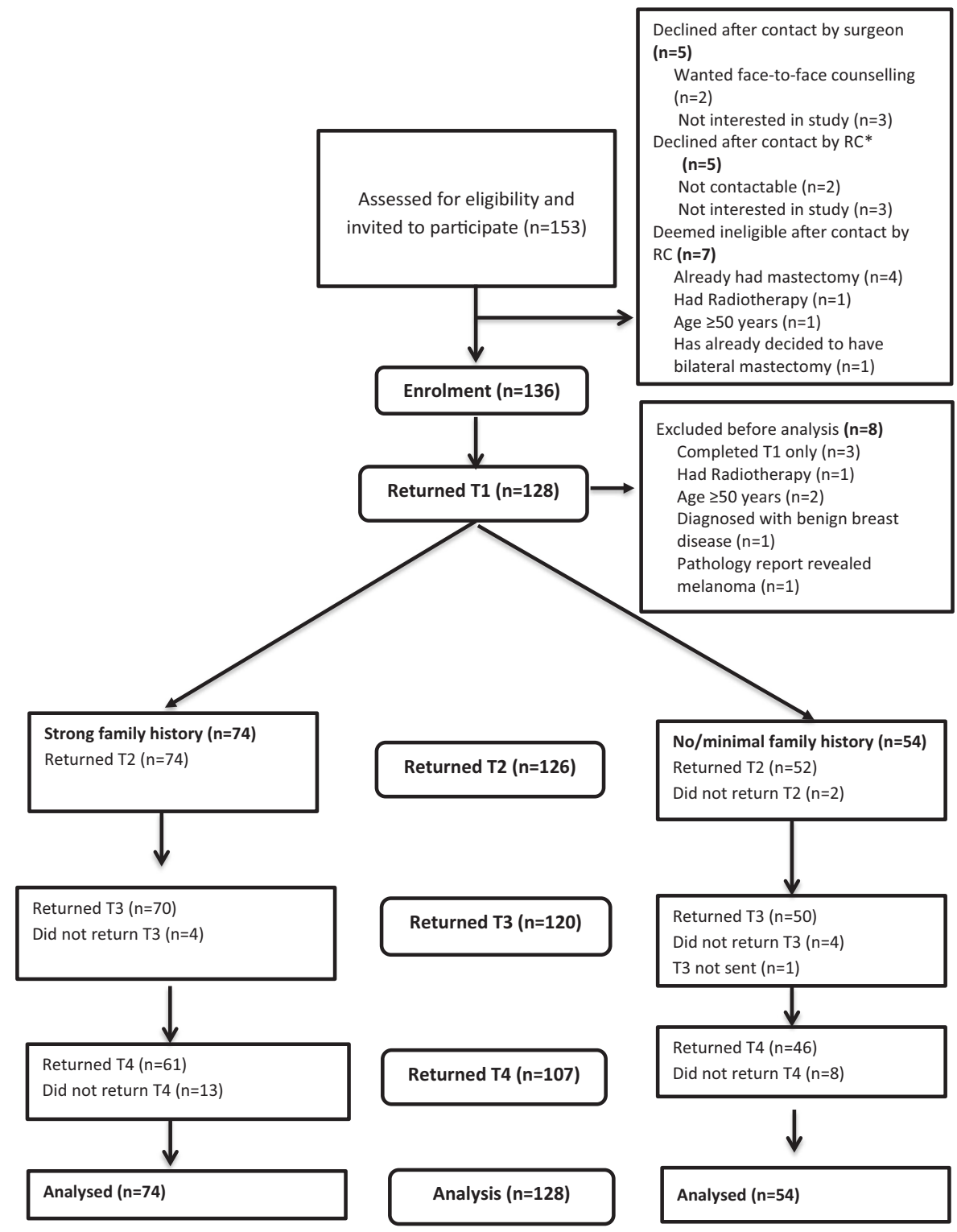

Legend: * RC= Research Co-Ordinator; $\mathrm{T} 1$-baseline questionnaire; $\mathrm{T} 2=$ within one week of receiving education; T3 $=2$ weeks post-disclosure of testing result; T4 = 12 months after study enrolment. between the two groups. Eighteen $(14.1 \%)$ women were found to harbour deleterious variants in $B R C A 1 / 2$ with the remaining $110(85.9 \%)$ having no such variant dentified. Of the 18 carriers of a disease-causing variant, nine (50.0\%) were recruited to the study on the basis of family cancer history, and six $(33.3 \%)$, two $(11.1 \%)$ and one $(5.6 \%)$ because of triplenegative pathology, bilateral cancer and Ashkenazi Jewish ancestry, respectively. There was cross-over between categories because surgeons were asked to indicate one eligibility criterion only.

Table 2 shows the mean scores and standard deviations for all psychological variables at the four assessment time points for $\mathrm{FH}+$ and $\mathrm{FH}$ - women, separately for carriers and non-carriers of a disease-causing variant.

\section{Psychological adjustment}

When averaged across variant status, FH- women reported significantly greater regret regarding undergoing TFGT than $\mathrm{FH}+$ women (estimated difference $=5.86, p=.019 ; 95 \%$ CI:1.03-10.71). A significant family history by variant status interaction ( $p=.023$ ) was followed by simple effects analysis, where it was revealed that, for women with no disease-causing variant found, there was no difference between $\mathrm{FH}+$ and $\mathrm{FH}-$ women (estimated difference $=$ $0.18, p=.931 ; 95 \% \mathrm{CI}:-4.24-3.88)$, whereas for those with a disease-causing variant found, $\mathrm{FH}-$ women reported significantly higher regret scores than $\mathrm{FH}+$ women (estimated difference $=11.55, p=.024 ; 95 \%$ CI: 2.80-20.30). 
Table 2 Magnitude of changes from baseline for psychological outcomes $(N=128)$

\begin{tabular}{|c|c|c|c|}
\hline Measure & $\begin{array}{l}\text { Carriers of pathogenic } \\
\text { variant } N=18\end{array}$ & $\begin{array}{l}\text { No pathogenic variant } \\
\text { found } N=110\end{array}$ & $\begin{array}{l}\text { Total } \\
N=128\end{array}$ \\
\hline Niedare & Mlean (SD) & & \\
\hline \multicolumn{4}{|c|}{ Breast-cancer-specific worry (T1) } \\
\hline Family history & $34.0(11.7)$ & $33.8(14.3)$ & $33.4(14.0)$ \\
\hline No/minimal family history & $38.1(15.2)$ & $31.0(14.6)$ & $32.2(14.8)$ \\
\hline \multicolumn{4}{|c|}{ Breast-cancer-specific worry (T2) } \\
\hline Family history & $31.3(12.7)$ & $29.2(16.2)$ & $29.5(15.7)$ \\
\hline No/minimal family history & $27.1(13.3)$ & $28.5(16.6)$ & $28.3(15.9)$ \\
\hline \multicolumn{4}{|c|}{ Breast-cancer-specific worry (T3) } \\
\hline Family history & $33.4(12.8)$ & $26.2(17.7)$ & $27.0(17.2)$ \\
\hline No/minimal family history & $33.6(14.6)$ & $27.2(15.6)$ & $28.2(15.5)$ \\
\hline \multicolumn{4}{|c|}{ Breast-cancer-specific worry (T4) } \\
\hline Family history & $21.5(15.2)$ & $22.9(15.5)$ & $22.7(15.3)$ \\
\hline No/minimal family history & $34.1(17.7)$ & $25.2(17.4)$ & $27.0(17.6)$ \\
\hline \multicolumn{4}{|l|}{$H A D S$-anxiety $(T 1)$} \\
\hline Family history & $9.0(3.9)$ & $8.9(4.6)$ & $8.9(4.5)$ \\
\hline No/minimal family history & $11.0(4.8)$ & $9.5(4.2)$ & $9.7(4.3)$ \\
\hline \multicolumn{4}{|l|}{$H A D S$-anxiety (T2) } \\
\hline Family history & $7.3(5.1)$ & $8.2(5.0)$ & $8.1(4.9)$ \\
\hline No/minimal family history & $6.3(3.4)$ & $8.4(4.7)$ & $8.0(4.5)$ \\
\hline \multicolumn{4}{|l|}{ HADS-anxiety (T3) } \\
\hline Family history & $7.9(3.5)$ & $6.9(4.7)$ & $7.0(4.6)$ \\
\hline No/minimal family history & $8.6(2.6)$ & $7.4(4.6)$ & $7.6(4.3)$ \\
\hline \multicolumn{4}{|l|}{ HADS-anxiety (T4) } \\
\hline Family history & $4.6(4.5)$ & $6.3(4.0)$ & $6.1(4.1)$ \\
\hline No/minimal family history & $8.6(5.2)$ & $7.6(4.3)$ & $7.8(4.5)$ \\
\hline \multicolumn{4}{|l|}{ HADS-depression (T1) } \\
\hline Family history & $6.2(4.6)$ & $5.2(4.2)$ & $5.4(4.2)$ \\
\hline No/minimal family history & $7.9(3.9)$ & $5.2(3.6)$ & $5.6(3.8)$ \\
\hline \multicolumn{4}{|l|}{ HADS-depression (T2) } \\
\hline Family history & $4.4(3.5)$ & $4.6(4.4)$ & $4.6(4.3)$ \\
\hline No/minimal family history & $5.3(2.6)$ & $5.1(4.1)$ & $5.2(3.8)$ \\
\hline \multicolumn{4}{|l|}{ HADS-depression (T3) } \\
\hline Family history & $6.9(4.4)$ & $4.2(3.8)$ & $4.5(3.9)$ \\
\hline No/minimal family history & $7.6(3.6)$ & $5.7(3.8)$ & $6.0(3.8)$ \\
\hline \multicolumn{4}{|l|}{ HADS-depression (T4) } \\
\hline Family history & $3.0(2.9)$ & $3.6(3.6)$ & $3.5(3.5)$ \\
\hline No/minimal family history & $4.8(3.2)$ & $4.6(4.1)$ & $4.7(3.9)$ \\
\hline \multicolumn{4}{|l|}{ Test-related distress (T3) } \\
\hline Family history & $12.5(6.3)$ & $2.9(4.6)$ & $4.0(5.7)$ \\
\hline No/minimal family history & $17.1(9.4)$ & $3.3(4.1)$ & $5.5(7.3)$ \\
\hline \multicolumn{4}{|l|}{ Test-related distress (T4) } \\
\hline Family history & $5.6(5.4)$ & $1.7(2.9)$ & $2.3(3.6)$ \\
\hline No/minimal family history & $12.7(9.5)$ & $2.6(6.1)$ & $4.6(7.9)$ \\
\hline \multicolumn{4}{|c|}{ Test-related positive experiences (T3) } \\
\hline Family history & $11.4(5.3)$ & $16.3(4.4)$ & $15.8(4.7)$ \\
\hline No/minimal family history & $8.3(4.2)$ & $16.5(4.5)$ & $15.2(5.4)$ \\
\hline
\end{tabular}


Table 2 (continued)

\begin{tabular}{|c|c|c|c|}
\hline & $\begin{array}{l}\text { Carriers of pathogenic } \\
\text { variant } N=18\end{array}$ & $\begin{array}{l}\text { No pathogenic variant } \\
\text { found } N=110\end{array}$ & $\begin{array}{l}\text { Total } \\
N=128\end{array}$ \\
\hline Measure & Mean (SD) & Mean (SD) & Mean (SD) \\
\hline \multicolumn{4}{|c|}{ Test-related positive experiences (T4) } \\
\hline Family history & $11.0(2.3)$ & $14.5(6.6)$ & $14.1(6.3)$ \\
\hline No/minimal family history & $7.2(3.3)$ & $14.7(5.6)$ & $13.2(6.0)$ \\
\hline \multicolumn{4}{|c|}{ Decisional regret TFGT Choice (T4) } \\
\hline Family history & $21.0(2.8)$ & $25.7(8.9)$ & $25.0(8.5)$ \\
\hline No/minimal family history & $31.6(10.1)$ & $25.0(9.5)$ & $26.3(9.9)$ \\
\hline \multicolumn{4}{|c|}{ Decisional regret BM Choice (T4) } \\
\hline Family history & $22.5(7.1)$ & $33.5(14.1)$ & $32.1(13.8)$ \\
\hline No/minimal family history & $35.1(16.5)$ & $35.5(13.1)$ & $35.4(13.6)$ \\
\hline \multicolumn{4}{|c|}{ Decisional regret RRBSO Choice (T4) } \\
\hline Family history & $30.0(13.2)$ & $38.9(17.8)$ & $37.7(17.5)$ \\
\hline No/minimal family history & $48.0(16.4)$ & $41.2(16.1)$ & $42.4(16.2)$ \\
\hline
\end{tabular}

HADS Hospital Anxiety and Depression Scale. T1 Baseline, T2 2-weeks post-education, T3 2 weeks post-testing, T4 12 months post-enrolment

Figures 2-4 show time point simple effects for all psychological measures. When averaged across family history and variant status, there was a significant tendency for breast-cancer-specific worry to decrease over time. Compared to baseline, worry was an estimated 5.19 unstandardised units lower at post-education $(p \leq .001 ; 95 \%$ CI: 2.34-8.04), 5.10 units lower at 2-weeks post-results $(p=.007 ; 95 \%$ CI: $1.40-8.79)$, and 8.00 units lower at $12-$ months post-enrolment ( $p \leq .001$; 95\% CI: 3.89-12.12).

When averaged across family history and variant status, HADS Anxiety was significantly lower at post-education (2.07 units; 95\% CI: 1.30-2.83), 2-weeks post-results (2.14 units; 95\% CI: 1.18-3.10), and 12-months post-enrolment (2.88 units; $95 \%$ CI: $1.85-3.92$ ) compared to baseline (all $p<.001$ ). However, there were significant interactions between time point and variant status and time point and family history. Simple effect comparisons revealed that the decrease in anxiety from baseline to post-education was 2.20 units greater for carriers compared to non-carriers ( $p=.005 ; 95 \%$ CI: $0.66-3.73)$ and that the decrease from post-education to 2-weeks post-results was 2.23 units greater for non-carriers than carriers $(p=.006 ; 95 \% \mathrm{CI}$ : $0.63-3.82$ ). In women with a family history of cancer, the decrease in anxiety from 1-week post-education to one-year follow-up was 1.83 units greater $(p=.011 ; 95 \%$ CI: $0.43-3.23$ ) and from post-results to 1 -year follow-up it was 1.51 units greater $(p=.013 ; 95 \% \mathrm{CI}: 0.33-2.69)$ than in women with no family history.

When averaged across family history and variant status, HADS depression was 1.27 units lower post-education $(p<.001$; 95\% CI: $0.53-2.01)$ and 2.14 units lower 12months post-enrolment $(p<.001 ; 95 \%$ CI: 1.17-3.11)

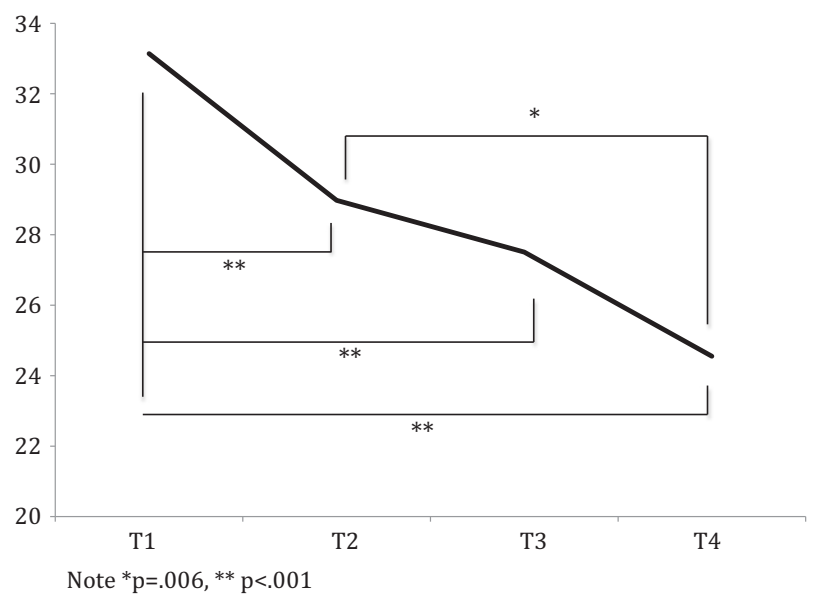

Fig. 2 Time point simple effects for Impact of Event Scale scores Note $* p=.006, * * p<.001$

compared to baseline. Furthermore, depression was 1.90 units lower at 1 -year post-enrolment than at 2 -weeks postresults $(p<.001 ; 95 \%$ CI: $1.11-2.69)$ and 1.03 units lower at 2 -weeks post-results than at post-education $(p=.008$; 95\% CI: $0.26-1.80)$. We found a significant time point by variant status interaction with follow-up comparisons that revealed that carriers of a $B R C A 1 / 2$ pathogenic variant had a 1.79-unit greater decrease from baseline to posteducation $(p=.019 ; 95 \%$ CI: $0.30-3.28)$, a 1.89 -unit greater decrease from post-education to post-results $(p=.020 ; 95 \%$ CI: $0.30-3.48)$, but a 1.90 -unit greater increase in depression from post-education to post-results ( $p=.016$; 95\% CI: 0.36-3.45) than non-carriers. However, by 12 -months post-enrolment there were no significant 


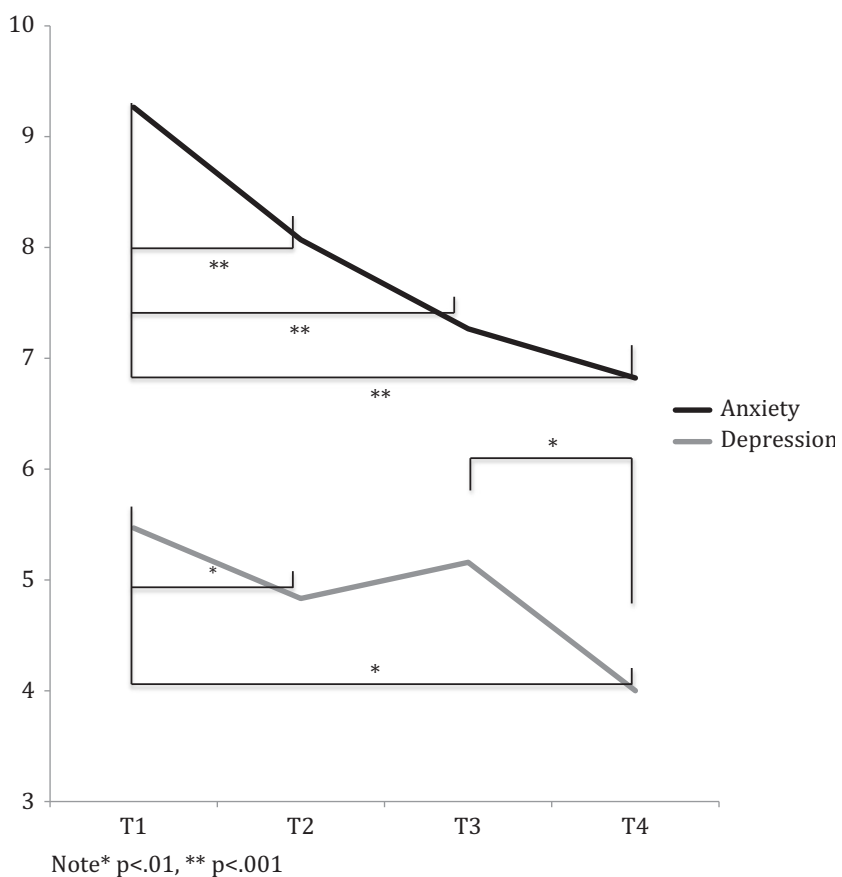

Fig. 3 Time point simple effects for Hospital Anxiety and Depression Scale scores Note $* p<.01, * * p<.001$

differences in depression found between carriers and noncarriers $(p=.98)$.

There was a significant main effect of time on test-related distress, with participants' distress an estimated 2.8 units lower at 12-months post-enrolment than post-results $(p<.001 ; 95 \%$ CI: 1.51-4.25). There was also a significant main effect of variant status, with test-related distress an estimated 9.37 points higher for carriers compared to non-carriers $(p<.001 ; 95 \%$ CI: 7.05-11.68) when averaged across family history and time. There was also a significant main effect of family history with $\mathrm{FH}+$ women showing test-related distress 3.19 units lower than FHwomen, averaged across time and variant status $(p=.008$, CI: 0.85-5.15). Simple effects comparisons revealed that carriers of a disease-causing variant had significantly higher test-related distress than non-carriers at both postresults (estimated difference $=11.45, p<.001,95 \%$ CI: $8.78-14.12$ ) and 12-months post-enrolment (estimated difference $=7.29, p<.001,95 \%$ CI: 4.56-10.00) but also had a 4.16-unit greater decrease in distress from post-results to 12-months post-enrolment compared to non-carriers ( $p=.004 ; 95 \%$ CI: 1.41-6.92). A significant family history by variant status interaction $(p=.046)$ qualified that, for women with no disease-causing variant found, there was a non-significant difference between $\mathrm{FH}+$ and $\mathrm{FH}-$ women of 0.8 units $(p=.403 ; 95 \%$ CI: $1.07-2.68)$, whereas for those with a variant found, FH- women reported significantly higher test-related distress scores than $\mathrm{FH}+$

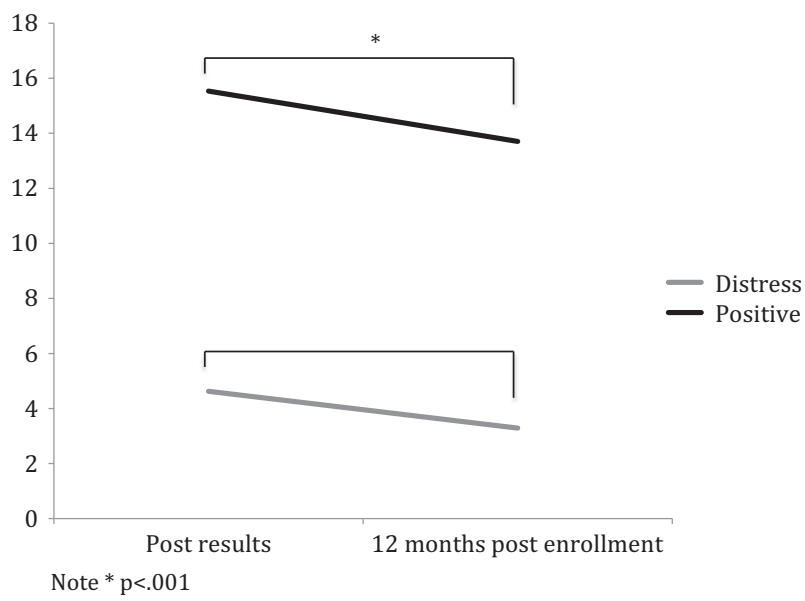

Fig. 4 Time point simple effects for Test-Related Positive and Experiences and Distress scoresNote $* p<.001$

women (estimated difference $=5.56, p=.012 ; 95 \%$ CI: 1.31-9.81).

Test-related positive experiences were rated significantly higher at post-results compared to 12-months post-enrolment (estimated difference 1.50, $p=.036 ; 95 \% \mathrm{CI}$ : $0.12-2.89$; 95\% CI: 0.12-2.89). Averaged across time points and family history, non-carriers had significantly more test-related positive experiences compared to carriers (estimated difference $=6.11, p<.001 ; 95 \%$ CI: 3.75-8.48).

\section{Surgical decision-making}

Due to low cell numbers, it was not possible to include the variant status by family history interaction for the regression on surgical uptake. For decisional regret regarding surgery analyses, it was of interest to include surgical decisions as covariates, but again low cell numbers prevented reasonable comparisons. Tables 3 and 4 show uptake of BM and RRBSO by variant status, separately for $\mathrm{FH}+$ and $\mathrm{FH}-$ women.

\section{Bilateral mastectomy}

At 12-months post-enrolment, 44 (34.4\%) women had had a BM (41 self-reported, and three as per medical records). A further 10 (7.8\% of 128) of the women who completed the 12-month post-enrolment questionnaire reported intending to have a BM. Carriers of a disease-causing variant (16, $88.9 \%$ ) were significantly more likely to have had a BM than women without an identified variant $(28,25.5 \%)$, with $\quad \mathrm{OR}=52.41 \quad(p<.001 ; \quad 95 \% \quad$ CI: 6.04-454.94); there were no significant differences in uptake of BM between $\mathrm{FH}+$ women $(23,31.1 \%)$ and $\mathrm{FH}-$ women $(21$, $38.9 \%), \quad(\mathrm{OR}=0.50, \quad p=.190 ; \quad 95 \% \quad \mathrm{CI}: \quad 0.18-1.41)$; adjusting for age, the original study randomisation and 
Table 3 Uptake of bilateral mastectomy by variant status separately for women with a strong and no/minimal family history

\begin{tabular}{|c|c|c|c|c|c|c|c|c|c|c|c|c|}
\hline \multirow[t]{3}{*}{ Variant status } & \multicolumn{6}{|c|}{$\begin{array}{l}\text { Number of women who had BM within } 12 \text { months since } \\
\text { enrolment }(N=128)^{\mathrm{a}}\end{array}$} & \multicolumn{6}{|c|}{$\begin{array}{l}\text { Number of women who had not had a BM within } 12 \text { months } \\
\text { but reported intention to undergo BM }(N=66)^{\text {b,c }}\end{array}$} \\
\hline & \multicolumn{2}{|c|}{ Family history } & \multicolumn{2}{|c|}{ No family history } & \multicolumn{2}{|l|}{ Total } & \multicolumn{2}{|c|}{ Family history } & \multicolumn{2}{|c|}{$\begin{array}{l}\text { No family } \\
\text { history }\end{array}$} & \multicolumn{2}{|l|}{ Total } \\
\hline & Tot. $N$ & $N(\%)$ & Tot. $N$ & $N(\%)$ & Tot. $N$ & $N(\%)$ & Tot. $N$ & $N(\%)$ & Tot. $N$ & $N(\%)$ & Tot. $N$ & $N(\%)$ \\
\hline Variant carriers & 9 & $7(77.8)$ & 9 & $9(100.0)$ & 18 & $16(88.9)$ & 1 & $1(100.0)$ & 0 & $0(0)$ & 1 & $1(100.0)$ \\
\hline No variant found & 65 & $16(24.6)$ & 45 & $12(26.7)$ & 110 & $28(25.5)$ & 39 & $9(23.1)$ & 26 & $0(0)$ & 65 & $9(13.8)$ \\
\hline Total & 74 & $23(31.1)$ & 54 & $21(38.9)$ & 128 & $44(34.4)$ & 40 & $10(25.0)$ & 26 & $0(0.0)$ & 66 & $10(15.2)$ \\
\hline
\end{tabular}

Variant carrier is the carrier of a $B R C A 1$ or $B R C A 2$ pathogenic variant

Tot. $N$ Total $N$ separately for women with a strong family history and no/minimal family history. $B M$ bilateral mastectomy

${ }^{a}$ Self-reported as at 12-months post-enrolment questionnaire and as per medical records for women with missing self-report data

${ }^{\mathrm{b}}$ Self-reported as at 12-months post-enrolment questionnaire

${ }^{\mathrm{c}}$ Data on intention to undergo BM are missing for 18 women

prognostic status. Decisional regret regarding surgical choice was assessed for all women regardless of whether or not they had surgery. For decisional regret regarding whether or not a woman had undergone a BM, averaged across variant status, $\mathrm{FH}-$ women reported more regret than $\mathrm{FH}+$ women (estimated difference $=8.65, p=.022 ; 95 \% \mathrm{CI}$ : 1.36-15.96).

\section{Risk-reducing bilateral salpingo-oophorectomy}

At 12-months post-enrolment 17 (13.4\%) women had undergone a RRBSO (all self-reported). A further 22 women with 12-months self-report data available reported intending to have an RRBSO. At the 12-months follow-up, of the 18 carriers included in this sample, five (all with a strong family history) had already had a RRBSO (28\%), and 11 of those who had not had a RRBSO intended to have a RRBSO, and only one did not. Controlling for family history, study arm, and prognostic status, women aged $>40$ years were more likely to have undergone a RRBSO than those aged $\leq 40$ years $(\mathrm{OR}=6.84, p=.022 ; 95 \% \mathrm{CI}$ : 1.31-35.74). Of the nine $\mathrm{FH}+$ carriers of a pathogenic variant, five had already had a RRBSO at 12-months, three intended to have a RRBSO, and self-report data on intention were missing for one woman. Of the nine $\mathrm{FH}$ - carriers of a pathogenic variant, none had had a RRBSO at 12-months but eight intended to have a RRBSO, and one FH - carrier did not. Five of the carriers also had a family history of ovarian cancer; of these one had had a RRBSO (20.0\%) and four $(80.0 \%)$ intended to have a RRBSO. When controlling for family history, study arm, and prognostic status, carriers of a pathogenic variant $(5,27.8 \%)$ were significantly more likely to have undergone RRBSO than non-carriers (12, $10.9 \%),(\mathrm{OR}=6.06, p=.022 ; 95 \% \mathrm{CI}: 1.30-28.22)$. When controlling for family history, study arm, and prognostic status, there was no statistically significant difference between $\mathrm{FH}+$ women $(14,19.2 \%)$ and $\mathrm{FH}-$ women (3, $5.6 \%)$ in uptake of $\mathrm{RRBSO}(\mathrm{OR}=3.34, p=.093,95 \% \mathrm{CI}$ : 0.82-13.59).

For decisional regret regarding choice to have an RRBSO, averaged across variant status, FH- women reported more regret than $\mathrm{FH}+$ women (estimated difference $=11.67, p=.016 ; 95 \%$ CI: 2.38-20.96).

\section{Discussion}

These results suggest that women with breast cancer who are referred for TFGT and do not have a strong family history report poorer adjustment up to 12 months after diagnosis. Specifically, we found that women without a family history had lower decreases in anxiety a year after diagnosis and reported more regret regarding their choice whether or not to have BM and an RRBSO. Women without a family history who carried a pathogenic variant also reported greater regret regarding the choice to undergo TFGT, and greater test-related distress.

These findings indicate that women without a strong family history are more vulnerable to psychological sequelae of decision-making regarding surgery at this time in their lives, and that this group may require additional counselling to facilitate informed decisions. Given the higher regret regarding TFGT choice and test-related distress found in carriers of a disease-causing variant without a family history, these women may require additional followup counselling to facilitate psychological adjustment to their positive variant results, extra support in making surgical decisions, and counselling about how best to communicate results to family members. In particular, carriers with no/ minimal family history may benefit from more formal 


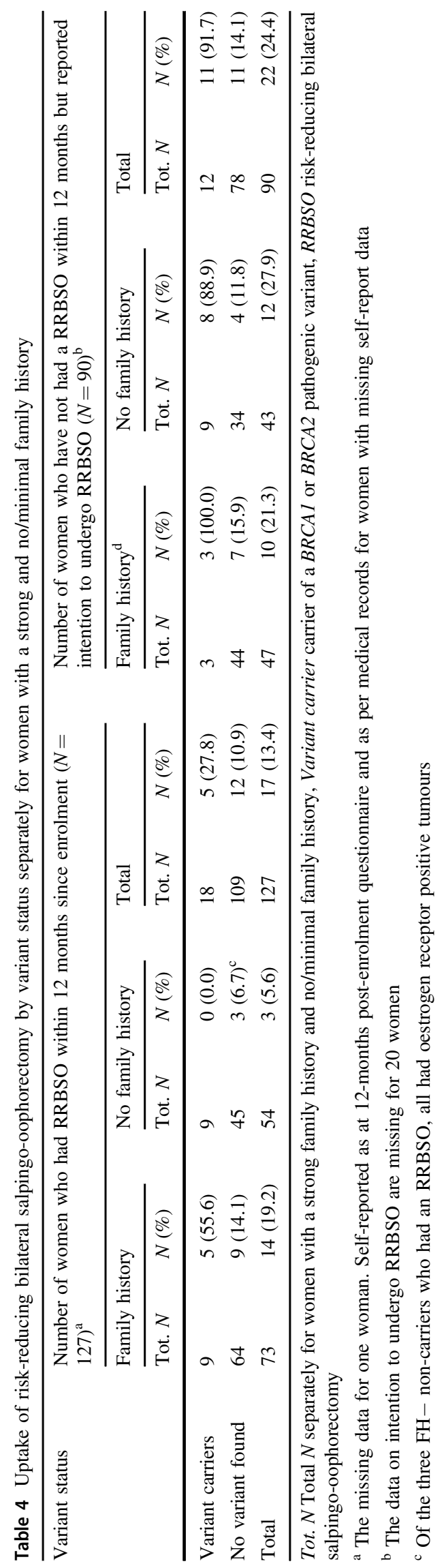

follow-up at the time of completion of cancer treatment. These data support the findings of our qualitative study of women offered TFGT following a new diagnosis of breast cancer [29]. Here it was found that long-term adjustment was hindered by a sense of isolation in women with no family history, and considerable support deficits in these women were observed [29].

The study demonstrated that carriers of a diseasecausing variant were significantly more likely to have a BM than women without a variant, with uptake rates of 89 and $26 \%$, respectively. These data confirm results from similar studies, which indicate high uptake rates amongst carriers [30]. Findings underscore that women value the certainty afforded by a positive variant result and indicate that it is the most critical variable influencing women's decision-making regarding BM. Reassuringly, at the 12-months follow-up, of the 18 carriers included in this sample, five (all with a strong family history) had already had a RRBSO (28\%), 11 of those who had not had a RRBSO intended to have a RRBSO, and only one did not. These findings indicate that almost all carriers of a diseasecausing variant understand the implications of their results and translate or intend to translate them into preventative behaviours. It is positive to note that the greater reported regret around surgical decisions in women without a family history did not appear to translate to a decision not to undergo BM or RRBSO.

The strengths of this study include a high follow-up rate at 12 months (84\%) and lack of differences between those lost to follow-up and those retained; surgical outcome data on BM were still available from file review for all of those lost to follow-up. All participants in this study opted to receive their TFGT results, and it is, therefore, likely that participants were self-selected for interest in genetic testing and may not be fully representative of the larger group of younger women newly diagnosed with breast cancer. However, the study was not designed to measure uptake of TFGT amongst this larger group, and other studies have already documented uptake rates, e.g., amongst consecutively recruited samples [15]. Nevertheless, study findings are generalisable to those women most likely to take up TFGT when offered. The majority of women $(94.5 \%)$ reported speaking mostly English at home and thus generalisability to minorities is limited. The number of disease-causing variant carriers included in the study was small, and the study may have been underpowered to detect differences by variant status in important outcomes such as regret after receipt of results, and to detect interactions with family history status in some circumstances. This reduced our ability to determine whether all family history effects were driven by carriers of a disease-causing variant, or whether women without a known family history who did not receive results, 
indicating presence of a variant were also experiencing additional psychological difficulties.

Acknowledgements We thank the patients who generously participated in this study. We also gratefully acknowledge the assistance of the staff who were involved at each site. Thank you also to Mariana Souza for research assistance. We also acknowledge the support and endorsement of this project by the Psycho-oncology Cooperative Research Group (PoCoG). This trial was funded by a Priority-Driven Collaborative Research Grant, which was jointly supported by Cancer Australia, Cancer Council Australia and the National Breast Cancer Foundation (ID 630405). Bettina Meiser was supported by a Career Development Fellowship Award Level 2 (ID 1003921) from the National Health and Medical Research Council (NHMRC) of Australia and an NHMRC Senior Research Fellowship Level B (ID 1078523). Michelle Peate is currently supported by a National Breast Cancer Foundation Early Career Fellowship (ECF-15-005).

TFGT Collaborative Group The additional members of the Treatment FocusedGenetic Testing Collaborative Group are: Cabrini Health Melbourne (P. Gregory, L.Lipton, L. McKay, J. Senior); Department of Medical Oncology, Prince of WalesHospital (P. Crowe, A. Matthews, G. Neil, A. Parasyn, D. Thomson); HereditaryCancer Clinic, Prince of Wales Hospital, Sydney (J. Duffy, L. Andrews, J. Gale); Monash Medical Centre, Melbourne (J. Fox, S. Hart, C. Smythe, M. White);Nambour Hospital, Nambour (L. Creighton, J. D'arcy, S. Grieve, E. Secomb); PeterMacCallum Cancer Centre, Melbourne (M. Henderson, J. O’Brien, C. Poliness);Royal Brisbane Hospital (A. Hattam, R. Susman, O. Ung,); Royal North ShoreHospital, Sydney (R. Dickson, K. Moore); St George Hospital, Sydney (P. Bastick, S.Inder, J. Lynch, P. Schwartz, R. Zia); The Poche Centre, Sydney (C. Mak, K. Snook,A. Spillane); University of Melbourne (J. Hopper); Westmead Hospital, Sydney (M.Bowman, D. Cheung, S. Edirimanne, E. Edwards, E. Elder, J. French, D. Moon).

\section{Compliance with ethical standards}

Conflict of interest BM, KT and MG have remunerated consultant roles with the company Astrazeneca with respect to unrelated projects. The remaining authors declare that they have no competing intersts.

\section{References}

1. Trainer A, Lewis C, Tucker K, Meiser B, Friedlander M, Ward R. Treatment-focused genetic assessment in breast cancer - the evolving role of the familial cancer services. Nat Rev Clin Oncol. 2010;7:708-17.

2. Metcalfe K, Lynch $\mathrm{H}$, Ghadirian $\mathrm{P}$, et al. Risk of ipsilateral breast cancer in BRCA1 and BRCA2 mutation carriers. Breast Cancer Res Tr. 2011;127:287-96.

3. van den Broek A, van't Veer L, Hooning M, et al. Impact of age at primary breast cancer on contralateral breast cancer risk in BRCA1/2 mutation carriers. J Clin Oncol. 2015;34:409-18.

4. Metcalfe K, Gershman S, Ghadirian P, et al. Contralateral mastectomy and survival after breast cancer in carriers of BRCA1 and BRCA2 mutations: retrospective analysis. BMJ. 2014;348:226.

5. Evans D, Ingham S, Baildam A, et al. Contralateral mastectomy improves survival in women with BRCA1/2-associated breast cancer. Breast Cancer Res Treat. 2013;140:135-42.

6. Rebbeck T, Kauff N, Domchek S, Meta-analysis of risk reduction estimates associated with risk-reducing salpingooophorectomy in
BRCA1 or BRCA2 mutation carriers. J Natl Cancer Inst. 2009;101:80-87.

7. Kotsopoulos J, Huzarski T, Gronwald J, et al. Bilateral oophorectomy and breastcancer risk in BRCA1 and BRCA2 mutation carriers. J Natl Cancer Inst. 2017;109:djw177

8. Telli M, Jensen K, Vinayak S, et al. Phase II study of gemcitabine, carboplatin, and iniparib as neoadjuvant therapy for triplenegative and BRCA1/2 mutation-associated breast cancer with assessment of a tumor-based measure of genomic instability: PrECOG 0105. J Clin Oncol. 2015;33:1895-901.

9. Moller P, Hagen A, Apold J, et al. Genetic epidemiology of BRCA mutations - family history detects less than $50 \%$ of the mutation carriers. Eur J Cancer. 2007;43:1713-7.

10. Fackenthal J, Olopade O. Breast cancer risk associated with BRCA1 and BRCA2 in diverse populations. Nat Rev Cancer. 2007;7:937-48.

11. Bradbury A, Patrick-Miller L, Long J, et al. Development of a tiered and binned genetic counseling model for informed consent in the era of multiplex testing for cancer susceptibility. Genet Med. 2015;17:485-92.

12. Hilgart J, Coles B, Iredale R. Cancer genetic risk assessment for individuals at risk of familial breast cancer. Cochrane Database Syst Rev. 2012;2:CD003721.

13. Hamilton JG, Lobel M, Moyer A. Emotional distress following genetic testing for hereditary breast and ovarian cancer: A metaanalytic review. Health Psychol. 2009;28:510-8.

14. Schlich-Bakker KJ, Warlam-Rodenhuis CC, van Echtelt $\mathrm{J}$, van den Bout J, Ausems MG, ten Kroode HF. Short term psychological distress in patients actively approached for genetic counselling after diagnosis of breast cancer. Eur J Cancer. 2006;42: 2722-8.

15. Schlich-Bakker KJ, Ausems MG, Schipper M, Ten Kroode HF, Warlam-Rodenhuis CC, BRCA1/2 mutation testing in breast cancer patients: a prospective study of the long-term psychological impact of approach during adjuvant radiotherapy. Breast Cancer Res Treat. 2008;109:507-14.

16. Shkedi-Rafid S, Gabai-Kapara E, Grinshpun-Cohen J, LevyLahad E. BRCA genetic testing of individuals from families with low prevalence of cancer: experiences of carriers and implications for population screening. Genet Med. 2012; 14:688-94.

17. Manchanda R, Loggenberg K, Sanderson S, et al. Population testing for cancer predisposing BRCA1/BRCA2 mutations in the Ashkenazi-Jewish Community: a randomized controlled trial. J Natl Cancer Inst. 2015;107:379.

18. Marteau TM, Croyle RT. Psychological responses to genetic testing. Br Med J. 1998;316:693-6.

19. Watts K, Meiser B, Mitchell G, et al. How should we discuss genetic testing with women newly diagnosed with breast cancer? Design and implementation of a randomized controlled trial of two models of delivering education about treatment-focused genetic testing to younger women newly diagnosed with breast cancer. BMC Cancer. 2012;12:320.

20. Quinn V, Meiser B, Kirk J, et al. Streamlined education is effective for women newly diagnosed with breast cancer considering genetic testing. Genet Med. 2017;19:448-56.

21. National Breast Cancer Centre. Advice about familial aspects of breast cancer and epithelial ovarian cancer: A guide for health professionals. Sydney: NHMRC National Breast and Ovarian Cancer Centre; 2010.

22. Thewes B, Meiser B, Hickie I. Psychometric properties of the Impact of Event Scale amongst women at increased risk for hereditary breast cancer. Psycho-Oncol. 2001;10:459-68.

23. Ibbotson $\mathrm{T}$, Maguire $\mathrm{P}$, Selby $\mathrm{P}$, Priestman $\mathrm{T}$, Wallace $\mathrm{L}$. Screening for anxiety and depression in cancer patients: the effects of disease and treatment. Eur J Cancer. 1994;30A:37-40. 
24. Cella D, Chang C, Peterman A, et al. A brief assessment of concerns associated with genetic testing for cancer: The multidimensional impact of cancer risk assessment (MICRA) questionnaire. Health Psychol. 2002;21:564-72.

25. Brehaut JC, O'Connor AM, Wood TJ, et al. Validation of a decision regret scale. Med Dec Mak. 2003;23:281-92.

26. Lannin D, Wang S. Are small breast cancers good because they are small or small because they are good? $\mathrm{N}$ Engl J Med. 2017;376:2286-91.

27. Singer J, Willett J. Applied longitudinal data analysis: Modeling change and event occurrence. New York: Oxford University Press; 2003.
28. Cancer Australia. Recommendations for the management of early breast cancer in women with an identified BRCA1 or BRCA2 gene mutation or at high risk of a gene mutation. in: Australia $\mathrm{C}$, editor. A clinical practive guideline. Canberra: Cancer Australia; 2014.

29. Meiser B, Quinn V, Gleeson M, et al. When knowledge of a heritable gene mutation comes out of the blue: treatment-focused genetic testing in women newly diagnosed with breast cancer. Eur J Hum Genet. 2016;24:1571-1523.

30. Schwartz M, Lerman $\mathrm{C}$, Brogan $\mathrm{B}$, et al. Impact of BRCA1/2 counseling and testing on newly diagnosed breast cancer patients. J Clin Oncol. 2004;22:1823-9.

\section{Affiliations}

Bettina Meiser ${ }^{1}$ - Veronica F. Quinn ${ }^{1} \cdot$ Gillian Mitchell ${ }^{2,3} \cdot$ Kathy Tucker $^{4} \cdot$ Kaaren J. Watts $^{1} \cdot$ Belinda Rahman $^{1}$. Michelle Peate $^{1,5} \cdot$ Christobel Saunders $^{6}$. Elizabeth Geelhoed ${ }^{7} \cdot$ Margaret Gleeson $^{8} \cdot$ Kristine Barlow-Stewart $^{9}$. Michael Field ${ }^{10} \cdot$ Marion Harris $^{11} \cdot$ Yoland C. Antill $^{12} \cdot$ Rachel Susman $^{13} \cdot$ Michael T. Bowen $^{14,15} \cdot$ Llew Mills $^{1}$. Judy Kirk ${ }^{16,17}$ for the TFGT Collaborative Group

1 Prince of Wales Clinical School, Faculty of Medicine, UNSW Sydney, Sydney, NSW 2052, Australia

2 Sir Peter MacCallum Dept of Oncology, Melbourne University, Melbourne, VIC 3010, Australia

3 Familial Cancer Centre, Peter MacCallum Cancer Centre, Melbourne, VIC 3002, Australia

4 Hereditary Cancer Clinic; Department of Medical Oncology, Prince of Wales Hospital, High Street, Randwick, NSW 2031, Australia

5 Obstetrics and Gynaecology, Royal Women's Hospital, University of Melbourne, Melbourne, VIC 3052, Australia

6 School of Surgery, University of Western Australia, Crawley, WA 6009, Australia

7 School of Population Health, University of Western Australia, Crawley, WA 6009, Australia

8 Hunter Family Cancer Service, Waratah, NSW 2298, Australia

9 Sydney Medical School-Northern, University of Sydney,
Sydney, NSW 2006, Australia

10 Royal North Shore Hospital, St Leonards, NSW 2065, Australia

11 Monash Health, Clayton, Victoria 3168, Australia

12 Familial Cancer Centre, Cabrini Health, Melbourne, VIC 3144, Australia

13 Genetic Health Queensland, Royal Brisbane and Women's Hospital, Herston, QLD 4029, Australia

14 School of Psychology, University of Sydney, Sydney, NSW 2006, Australia

15 Brain and Mind Centre, University of Sydney, Sydney, NSW 2006, Australia

16 Familial Cancer Service, Westmead Hospital, Hawkesbury Road, Westmead, NSW 2145, Australia

17 Centre for Cancer Research, Westmead Institute for Medical Research, University of Sydney, Westmead, NSW 2145, Australia 